\title{
Development of a fuzzy expert system for the control of glycemia in type 1 diabetic patients
}

\author{
Leonardo Nobile ${ }^{\mathrm{a}}$, Bartolomeo Cosenza ${ }^{\mathrm{a}}$, Marco Amato ${ }^{\mathrm{b}}$, Valentina \\ Guarnotta $^{\mathrm{b}}$, Carla Giordano ${ }^{\mathrm{b}}$, Aldo Galluzzo ${ }^{\mathrm{b}}$, Mosè Galluzzo ${ }^{\mathrm{a}}$ \\ ${ }^{a}$ Dipartimento di Ingegneria Industriale, Università degli Studi di Palermo, Viale delle \\ Scienze ed. 6, 90128, Palermo Italy \\ ${ }^{b}$ Dipartimento di Oncologia Sperimentale ed Applicazioni Cliniche, Sezione di \\ Endocrinologia, Università degli Studi di Palermo, Piazza delle Cliniche 2, 90127 \\ Palermo,Italy
}

\begin{abstract}
The paper describes the structure and the characteristics of an expert system that allows the optimization of postprandial glycemia in type 1 diabetic patients. The expert system is able to provide patients with the number of rapid insulin units that must be taken in order to keep the blood glucose level close to the omeostatic condition in the hours following a meal.
\end{abstract}

Keywords: fuzzy logic system, neuro fuzzy system, expert system, diabetes mellitus, glycemia control.

\section{Introduction}

Diabetes mellitus is a disease characterized by high blood glucose level as a result of either the body not producing enough insulin, or the body cells not properly responding to the insulin that is produced (insulin-resistance). There are many types of diabetes, the most common of which are type 1 diabetes and type 2 diabetes (World Health Organization, 2006). Type 1 diabetes results from the pancreatic beta-cells failure to produce insulin, and presently requires that patients inject themselves with insulin. Type 2 diabetes results from insulin resistance, a condition in which cells fail to properly use insulin, combined with an absolute or relative insulin deficiency. The expert system presented in this paper is concerned with type 1 diabetes only. Patients with type 1 diabetes mellitus need external insulin, subcutaneously injected, for their survival.

It is well known that patients with type 1 diabetes are exposed to frequent postprandial hyperglycemia or fasting and/or postprandial hypoglycemia.

Two different approaches have been proposed to determine the correct dose of insulin to be injected by diabetic patients. The most ambitious approach aims to provide an automatic system that works on a continuous basis starting from the measurement of the blood glucose, calculating the correct dose of insulin that is then injected by a pump (Bergman et al., 1979; Sorensen; 1985; Magni et al.,2009; Chee and Fernando, 2007) The second approach instead intends to offer an off line system, called bolus calculator, able to calculate the correct dose of insulin to be assumed on the occasion of a meal. Systems based on the first approach present main problems related to the measurement devices and pumps, but also to the choice of a suitable control algorithm. Model based 
control algorithms are the most promising ones but the development of reliable mathematical models of the involved metabolic processes is still on. The second approach seems to be able to lead to a more rapid, reliable and cheaper system. The system here described follows this approach.

Several systems have been already proposed. They differ for the variables used as inputs and outputs, the calculation algorithm and the validation method. Campos Delgado et al. (2006) proposed a system that works like a two loops off line control system. One control loop regulates the rapid insulin to be injected at meals time while the second loop works on a daily basis regulating the slow insulin. Their controller uses as input only the preprandial glycemia. The validation of the system has been carried out by simulation using a compartmental model. Mathews and Pelzer (2009) propose a bolus calculator that takes into account the energy of carbohydrates, the metabolic efficiency and the dependence on the person of the energy that is transformed in blood glucose. Shapira et al. (2010) take into account as well the carbohydrate content of the meal but to overcome the uncertainty of its determination they propose a decision support tool that is based on the optimization of insulin doses for carbohydrate ranges. Pankowska and Blazik (2010) propose a bolus calculator that considers proteins and lipids in addition to carbohydrates for two out of phase insulin injections that are justified by the different absorption time.

The expert system developed by the authors, to be used by type 1 diabetic patients in the period between two medical visits, takes into account the content of the meal in terms of carbohydrates, lipids and proteins but also considers the patient insulin sensitivity. The system is conceived to provide patients with the number of rapid insulin units that may assure optimal glycemia values after a meal. To this end data concerning the meal, the physical and clinical personal conditions, including pre-prandial glycemia, and the last insulin prescription of the doctor, must be supplied to the system. This works like an off line mixed feedback-feedforward controller based on the measurement of blood glucose level (the output variable) and the measurement of the type and amount of the food taken on (main disturbances). The necessity of handling the patients meal food data, that are inherently fuzzy, and modeling a very complex biological system, for which reliable mathematical models are not yet available, suggested the use of fuzzy and neuro-fuzzy techniques for the implementation of the off line system.

The development of the expert system was carried out on the basis of a set of clinical data specifically collected and consisted of three main phases: In the first phase a group of type 1 diabetic patients were involved, for a period of time, in recording data concerning their physical an clinical conditions, the type and amount of food taken on during their meals together with glycemia levels before and after them.

The second phase consisted of the development of the hierarchical fuzzy system. The rules and the membership functions of some fuzzy subsystems were chosen on the base of the knowledge and experience of doctors. Experimental data, provided by patients, were used for training and validating the neuro-fuzzy subsystem. The third phase consisted of testing the hierarchical fuzzy system with the experimental data.

\section{The expert system}

The expert system is constituted by three fuzzy systems and one adaptive neuro-fuzzy inference system (ANFIS) as shown in Fig. 1 and is characterized by a hierarchical structure. 


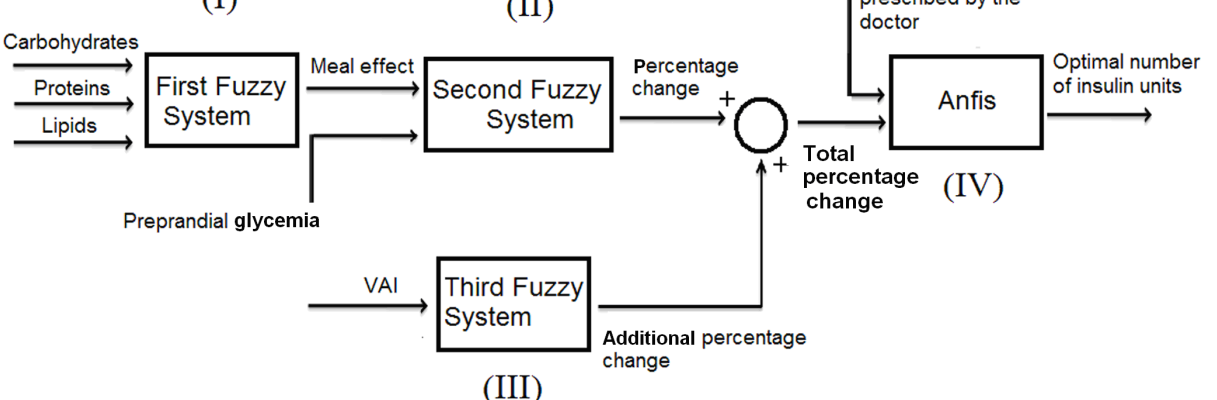

(I)

(III)

(II)

Fig.1. Block diagram of the Fuzzy System

The inputs of the fuzzy system are the type and the amount of food taken on during the meal (converted in amounts of carbohydrates, proteins and lipids), the preprandial glycemia, the number of rapid insulin units recommended by the therapy and the Visceral Adiposity Index (VAI), an index suggested by some of the authors (Amato et al., 2010) that estimates the patient insulin sensitivity. The VAI is gender specific, is calculated through the values of triglycerides, high density lipoprotein (HDL) and the patient body mass index, and has a good correlation with the euglycemic hyperinsulinemic Clamp test (DeFronzo, 1979), considered as the Gold standard for the insulin sensitivity estimation.

The first fuzzy system uses the Mamdani inference method (Mamdani, 1977) and is constituted by three inputs (Fig. 1) which are the amounts of carbohydrates, proteins and lipids assumed by the patient during the meal. Their values are evaluated by a calculation module that uses the information about the type and amount of food and the nutrition tables drawn by the Italian National Research Institute for Food and Nutrition (INRAN, 2010). The output is a parameter called "meal effect" that represents the effect that the meal has on the blood glucose concentration.

The second fuzzy system has two inputs and one output: the inputs are the "meal effect" (the output of the first fuzzy system) and the preprandial glycemia, a measurement that patients usually do before a meal, using a personal blood glucose meter.

The membership functions and the parameters for the "meal effect" are the same provided for the first system, while there are five membership functions for the preprandial glycemia. The output represents the percentage change of the number of rapid insulin units prescribed by doctors in the traditional therapy that should be applied as consequence of the meal effect and the preprandial glycemia value.

The third fuzzy system uses the Sugeno inference method (Sugeno and Kang, 1998). It has only one input and one output: the input is the VAI and the output is an additional percentage change of prescribed insulin units depending on the VAI.

The sum of the two percentage changes calculated by the second and the third system, constitutes one of the two inputs of an ANFIS system (Jang, 1993). The second input is the number of the rapid insulin units prescribed by the doctors in the traditional therapy. The output is the number of units able to maintain the concentration of glucose in the blood close to the homeostatic condition. The ANFIS structure is trained making use of a set of input-output data: the input data are constituted of the percentage changes calculated by the previous fuzzy systems and the insulin units originally prescribed by doctors; the output, i.e. the target, is the number of optimal units suggested by expert 
doctors on the base of the knowledge of the clinical state (preprandial glycemia, VIA) and the meal characteristics. All the characteristics of the fuzzy systems (membership functions, input and output ranges, rules) were established on the basis of the experience and knowledge of several doctors. The proposed system has been tested on a set of 158 cases, 120 used for the training 38 for the validation. The graph in Fig. 2 a) shows the number of cases with a given error (as percentage of total cases) versus the error produced by the expert system (as percentage of prescribed insulin units). Notice that the percentage of cases with zero error is about $65.2 \%$.
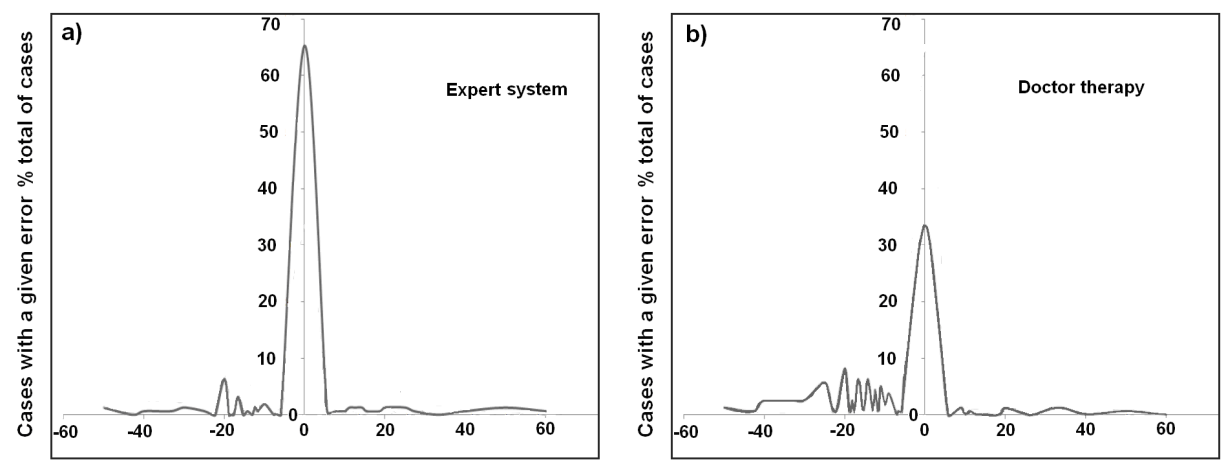

Fig. 2. a) Percentage of cases with a given error vs. \% error (percentage of prescribed insulin units) produced by the expert system. b) Percentage of cases with a given error vs. \% error (percentage of prescribed insulin units) when original prescribed insulin units are used.

Fig. 2 b) shows the analogue graph if the original doctor prescriptions are followed. Notice that the percentage of cases with zero error is much lower (about $33.5 \%$ ).

\section{Conclusions}

The results obtained with the proposed hierarchical fuzzy system are very encouraging. The expert system is in fact able to suggest the appropriate change of insulin units to be injected after a meal. The reference used for the evaluation of the expert system is the prescription of expert doctors based on the same input data. In almost all cases the system is able to suggest the same therapy change indicated by the doctors. The performance is even more appreciable if the comparison is made with the original doctor prescription.

The performance could probably improve if the physical activity after the meal is also considered. Another improvement, in particular in the first subsystem, could come from the use of type-2 fuzzy sets, that have a higher performance in dealing with uncertain data (Mendel 2001, Galluzzo and Cosenza, 2010), as the meal data.

The system could be tuned also for a specific patient through an off line further adaptation of the ANFIS module.

\section{References}

Amato, M., Giordano, C., Galia, M., Criscimanna, A., Vitabile, S., Midiri, M. and Galluzzo, A., 2010, Visceral Adiposity Index (VAI): a Reliable Indicator of Visceral Fat Function Associated with Cardiometabolic Risk, Diabetes Care, 33(4):920-2. 
Development of a fuzzy expert system for the control of glycemia in type 1 diabetic patients

Bergman, R. N., Ider, Y. Z., Bowden C. R. and Cobelli C., 1979, Quantitative estimation of insulin sensitivity, American Journal of Physiology, 236, 6, 667-677.

Campos-Delgado, D. U., Hernández-Ordoñez, M., Femat, R., and Gordillo-Moscoso, A., 2006, Fuzzy-Based Controller for Glucose Regulation in Type-1 Diabetic Patients by Subcutaneous Route. IEEE Transactions on biomedical engineering, 53 (11), 2201-2210.

Chee, F. and Fernando, T., 2007, Closed-Loop Control of Blood Glucose, Lecture Notes in Control and Information Sciences, Springer-Verlag, Berlin.

DeFronzo, R. A., Tobin, J. D. and Andres, R., 1979, Glucose clamp technique: a method for quantifying insulin secretion and resistance. Am. J. Physiol. Gastrointest. Liver Physiol., 237, G214-G223.

Galluzzo, M., Cosenza, B., 2010, Adaptive Type-2 Fuzzy Logic Control of a Bioreactor, Chemical Engineering Science, 65, 4208-4221.

INRAN, 2010, http://www.inran.it).

Jang, J. S. R., 1993, ANFIS: Adaptive-Network-based Fuzzy Inference Systems, IEEE Transactions on Systems, Man, and Cybernetics, 23, 3, 665-685.

Magni, L., Raimondo, D.M., Dalla Man, C., De Nicolao, G., Kovatchev, B. and Cobelli C., 2009, Model predicative Control of glucose concentration in type I diabetic patients: An in silico trial, Biomedical Signal Processing and Control, 4, 338-346.

Mamdani, E. H., 1977, Applications of fuzzy logic to approximate reasoning using linguistic synthesis, IEEE Transactions on Computers, 26, 12, 1182-1191.

Mathews, E. H. and Pelzer, R., 2009, A New Model to Estimate Bolus Insulin Need". Diabetes Technology \& Therapeutics, 11(12), 813-817.

Mendel, J. M., 2001, Uncertain Rule-Based Fuzzy Logic Systems: Introduction and New Directions, Upper Saddle River, NJ: Prentice-Hall.

Pankowska, E. and Blazik, M., 2010, Bolus calculator with nutrition database software, a new concept of prandial insulin programming for pump user, Journal of Diabetes Science and Technology, 4( 3), 571-576.

Shapira, G., Yodfat, O., HaCohen, A., Feigin, P. and Rubin R., 2010, Bolus Guide: A novel Insulin Bolus Dosing Decision Support Tool Based on Selection of Carbohydrate Ranges, Journal of Diabetes Science and Technology, 4 (4), 893-902.

Sorensen, J. T., 1985, A physiologic model of glucose metabolism in man and its use to design and assess improved insulin therapies for diabetes, Ph.D thesis, Dept. Chem. Eng., Massachusetts Institute of Technology, Cambridge.

Sugeno, M. and Kang, G. T, 1998, Structure identification of Fuzzy Model, Fuzzy sets and systems, 28,1, 15-33.

World Health Organization, 2006. Definition and diagnosis of diabetes mellitus and intermediate hyperglycemia. Report of a WHO/IDF Consultation. Geneva, World Health Organization. 